\title{
Analysis of Statistical Scatter in Charpy Impact Toughness
}

\author{
Yasuhito Takashima ${ }^{1,}$, Mitsuru Ohata ${ }^{1, b}$ and Fumiyoshi Minami ${ }^{1, c}$ \\ ${ }^{1}$ Division of Materials and Manufacturing Science, Graduate School of Engineering, \\ Osaka University,
}

2-1 Yamada-Oka, Suita, Osaka 565-0871, JAPAN,

atakasima@mapse.eng.osaka-u.ac.jp, bohata@mapse.eng.osaka-u.ac.jp, cminami@mapse.eng.osaka-u.ac.jp

\begin{abstract}
Keywords: Charpy absorbed energy, statistical distribution, weakest link theory, stress distribution, 3D FE analysis, brittle fracture.
\end{abstract}

\begin{abstract}
Charpy impact toughness values show large statistical scatter, particularly in the ductile-to-brittle transition temperature (DBTT) range. Although the statistical distribution of Charpy absorbed energy has not been clarified, critical values of the stress intensity factor, $J$-integral and crack-tip opening displacement (CTOD) at brittle fracture generally show the Weibull distribution with two or three parameters.

This study proposes a brittle fracture model, based on the weakest link theory, for evaluating the scatter in Charpy absorbed energy $K V$. The numerical results show that the amplitude of the opening stress fields ahead of the $\mathrm{V}$-notch at varying amounts of $K V$ are uniquely characterized as the square of the applied load. With these numerical results, the Weibull shape parameter of the statistical distribution of $K V$ is almost equal to 2 .

The proposed statistical model is verified through experimental results. It is found that the statistical distribution of $K V$ is characterized by a two-parameter Weibull distribution with the shape parameter of 2 under the condition of pure brittle fracture.
\end{abstract}

\section{Introduction}

The Charpy V-notch impact test is widely used for evaluating material toughness. It is well known that Charpy impact toughness values show large scatter, particularly in the ductile-to-brittle transition temperature (DBTT) range. The physical meaning of the statistical scatter of $K V$ values has not been clarified. Hirata et al. [1] and Kanazawa et al. [2] conducted a large number of Charpy tests on steel plates and reported that the distribution of $K V$ values is neither a normal distribution nor a log-normal distribution. It is difficult to clarify the type of statistical distribution of $K V$ by a statistical analysis of only the experimental data. Therefore, theoretical and analytical approaches to brittle fracture are necessary to investigate the statistical distribution.

However, critical values of the fracture mechanics parameters, such as the stress intensity factor, $J$-integral and crack-tip opening displacement (CTOD), denoted as $K_{\mathrm{c}}, J_{\mathrm{c}}$ and $\delta_{\mathrm{c}}$, respectively, generally show the Weibull distribution with two or three parameters at brittle fracture [3-5]. The Weibull distribution of fracture toughness is treated as the weakest link theory. It is known that the Weibull shape parameter of the fracture toughness distribution takes on a theoretical value of 4 in $K_{\mathrm{c}}$ and 2 in $J_{\mathrm{c}}$ and $\delta_{\mathrm{c}}$, as long as the small-scale yielding condition is satisfied [4, 5]. The singular stress field ahead of the crack tip controls the fracture toughness statistics with a constant Weibull slope.

In the present work, the statistical scatter in Charpy impact toughness is analyzed. The stress field ahead of the $\mathrm{V}$-notch in a Charpy specimen is investigated in terms of the stress singularity. Then, a brittle fracture model is proposed, based on the weakest link theory, for evaluating the statistical scatter in $K V$. The theoretical value for the Weibull slope in the case of the Charpy V-notch impact test is evaluated with the proposed model. The validity of this statistical model is discussed according to the experimental results obtained for structural steels and welds in other studies $[2,6]$. 


\section{Numerical Analysis of Three-dimensional Stress Field}

The stress fields near the V-notch of the Charpy specimen were numerically analyzed with a three-dimensional (3D) finite element (FE) code [Abaqus standard Ver. 6.12]. In this study, three types of procedures were employed: elastic stress analysis, elastic-plastic stress analysis, and quasi-static analysis considering the effect of the loading rate on strength.

Fig. 1 shows the Charpy V-notch specimen employed in the FE analysis. The specimen has the

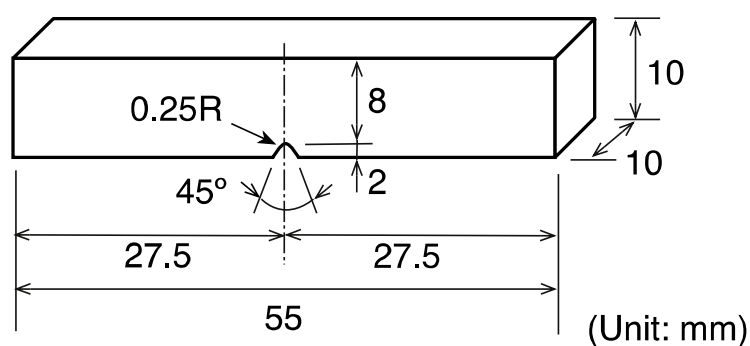

Fig. 1 Configuration of the V-notch Charpy specimen. standard $\mathrm{V}$-notch (depth $c=2 \mathrm{~mm}$ and root radius $\rho=0.25 \mathrm{~mm}$ ). The FE model is shown in Fig. 2. Due to the symmetry, only one-quarter of the specimen was modeled. The FE analysis used eight-noded elements with eight Gaussian integration points, and the minimum element size near the notch root was $0.05 \times 0.05 \times 0.2 \mathrm{~mm}$.

In the elastic-plastic FE analysis, hardening by the Swift type power law was used:

$$
\bar{\sigma}=\sigma_{\mathrm{Y}}\left\{1+\left(\bar{\varepsilon}_{\mathrm{p}} / \alpha\right)^{n}\right\}
$$

where $\bar{\sigma}$ and $\bar{\varepsilon}_{\mathrm{p}}$ are equivalent stress and equivalent plastic strain, respectively, $\sigma_{\mathrm{Y}}$ is the elastic limit (yield stress), and $n$ and $\alpha$ are material constants ( $n$ is a strain-hardening coefficient). The Young's modulus $E$ and Poisson's ratio $v$ are $206000 \mathrm{MPa}$ and 0.3 , respectively. The material properties of the elastic-plastic materials used in this study are shown in Table 1.

The stress field of the Charpy specimen subject to impact loading was analyzed by considering the strain rate effects on flow stress and the temperature rise during impact loading. The loading rate was $5 \mathrm{~m} / \mathrm{s}$ in accordance with the standard Charpy impact testing condition. The dependence of the yield stress and the tensile strength on the strain rate was evaluated as an empirical relationship [7]. The uniform elongation was assumed to be independent of the strain rate and the temperature.

Under the impact loading condition, high-speed straining generates heat adiabatically. It was assumed in the FE analysis that $90 \%$ of the plastic work is transferred to heat. The inertial effect during impact loading was eliminated by the quasi-static analysis. Based on the concept of transition time [8], the quasi-static analysis can be used to evaluate Charpy impact testing.

The value of $K V$ is evaluated as the

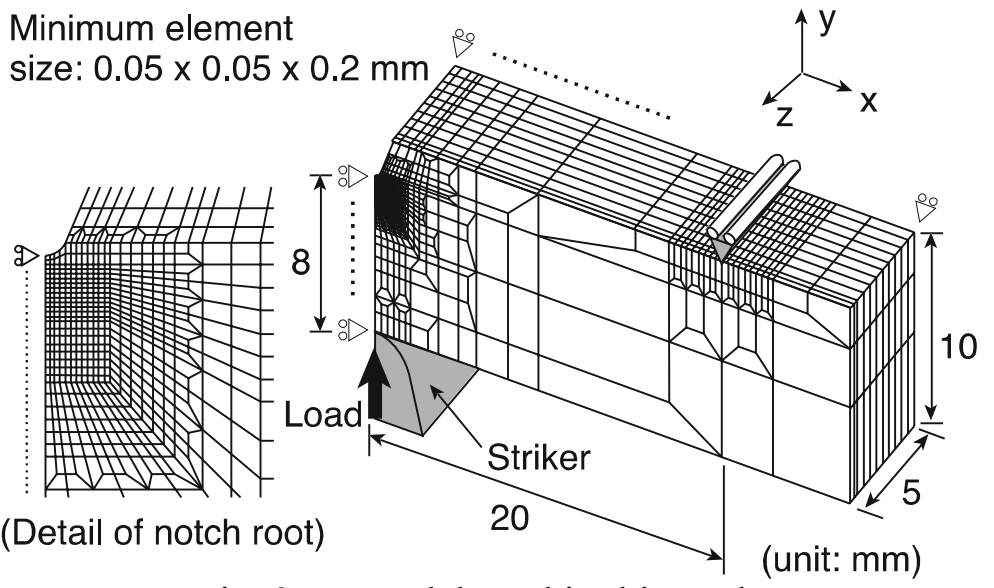

Fig. 2 FE model used in this study. amount of work done by the striker.

Table 1 Mechanical properties of elastic-plastic materials used in FE analysis.

\begin{tabular}{|l|c|c|c|c|c|c|}
\hline & $\sigma_{\mathrm{Y}}[\mathrm{MPa}]$ & $\sigma_{\mathrm{T}}[\mathrm{MPa}]$ & $\sigma_{\mathrm{Y}} / \sigma_{\mathrm{T}}$ & $\varepsilon_{\mathrm{T}}[\%]$ & $n$ & $\alpha$ \\
\hline Material A & 400 & 500 & 0.80 & 20 & 0.21489 & 0.03256 \\
\hline Material B & 800 & 842 & 0.95 & 7.0 & 0.09459 & 0.02693 \\
\hline
\end{tabular}

$\sigma_{\mathrm{Y}}$ : Yield stress, $\sigma_{\mathrm{T}}$ : Tensile strength, $\varepsilon_{\mathrm{T}}$ : Uniform elongation 


\section{Stress Field near V-notch in Charpy Specimen}

The linear elastic stress field near the $\mathrm{V}$-notch was investigated using the static 3D FE analysis.

Fig. 3 shows the opening stress distribution ahead of the V-notch in the linear elastic material. The numerical analysis indicates that the stress near the $\mathrm{V}$-notch is nearly inversely proportional to the square root of the distance $r$ from the notch root.

Hence, it can be assumed that $\sigma_{\mathrm{yy}}$ in the linear elastic material is given approximately by Eq. 2 :

$$
\sigma_{\mathrm{yy}}=\frac{k P /(B W)}{r^{1 / 2}} g(\theta)
$$

where $k$ is a constant, $P$ is the applied load, and $B$ and $W$ are the specimen thickness and width, respectively. Eq. 2 implies that the $1 / r^{1 / 2}$ singularity dominates the stress field ahead of the V-notch. From Eq. 2, the normalized stress field ahead of the V-notch can be written as follows:

$$
\frac{\sigma_{\mathrm{yy}}}{\sigma_{\mathrm{Y}}}=\frac{k}{r^{1 / 2}} \frac{P}{B W \sigma_{\mathrm{Y}}} g(\theta)=k\left\{r /\left(\frac{P}{B W \sigma_{\mathrm{Y}}}\right)^{2}\right\}^{-1 / 2} g(\theta)
$$

where $\sigma_{\mathrm{Y}}$ is the yield strength. In Eq. 3, $\left(P / \sigma_{\mathrm{Y}} B W\right)^{2}$ defines the amplitude of the linear elastic stress field.

The stress field near the V-notch in the elastic-plastic material was analyzed with the static and quasi-static 3D FE analyses. The opening stress distributions ahead of the $\mathrm{V}$-notch as a function of the parameter $x /\left(P / \sigma_{\mathrm{Y}} B W\right)^{2}$ for the Charpy specimen subject to static and impact loading are shown in Fig. 4 and Fig. 5, respectively. In the $K V$ range from $10 \mathrm{~J}$ to $50 \mathrm{~J}$, the stress distributions at varying amounts of $K V$ are uniquely described by Eq. 3 not only under the static loading condition but also the impact loading condition.

Hence, it is found that Eq. 3 is valid for evaluating the stress field in the Charpy V-notch

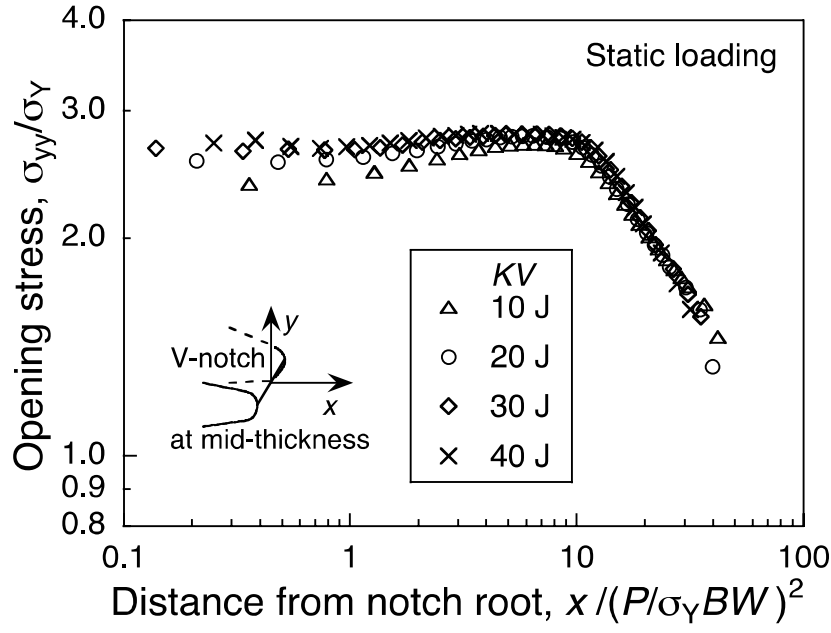

(a) $\sigma_{Y}=400 \mathrm{MPa}$

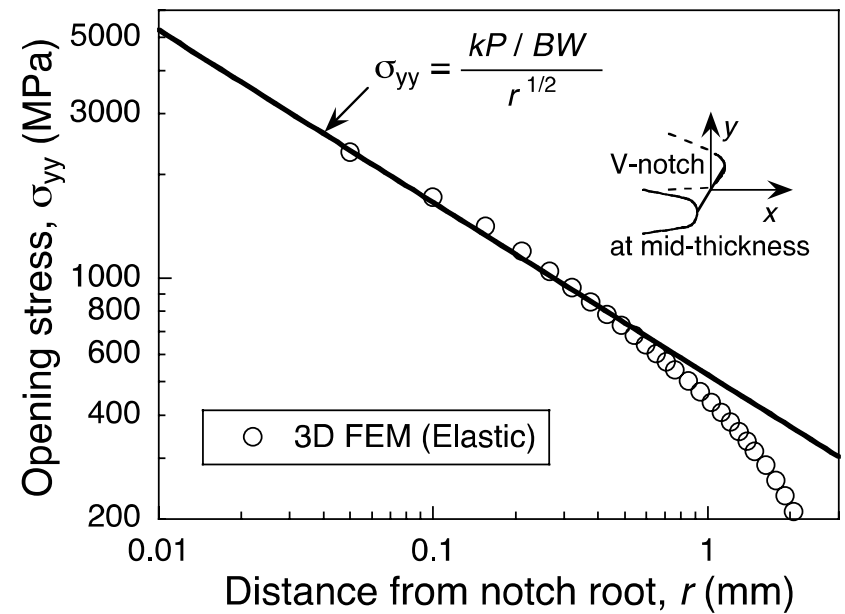

Fig. 3 Stress distribution near the $\mathrm{V}$-notch in the linear elastic material.

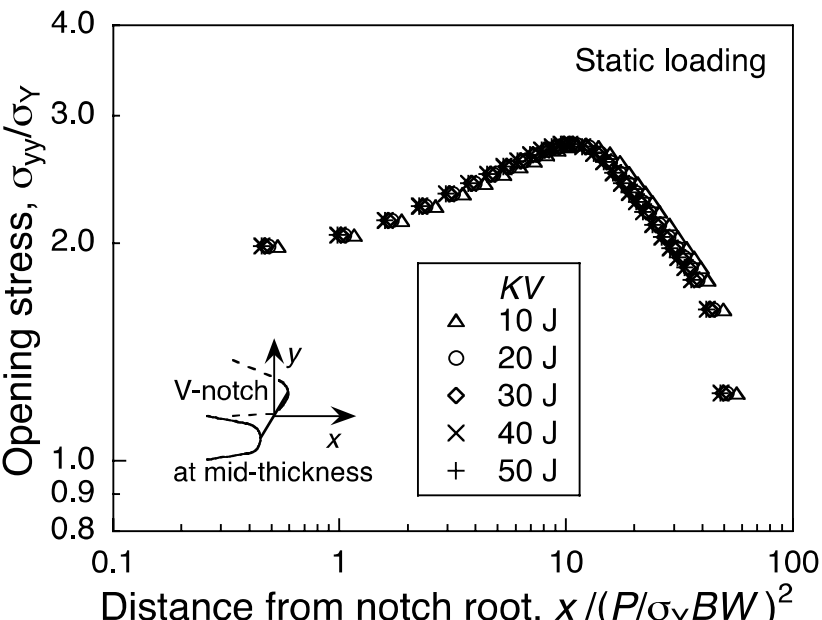

(b) $\sigma_{Y}=800 \mathrm{MPa}$

Fig. 4 Opening stress distribution near the V-notch in the Charpy specimen.

Subjected static loading as a function of the parameter $x /\left(P / \sigma_{\mathrm{Y}} B W\right)^{2}$. 


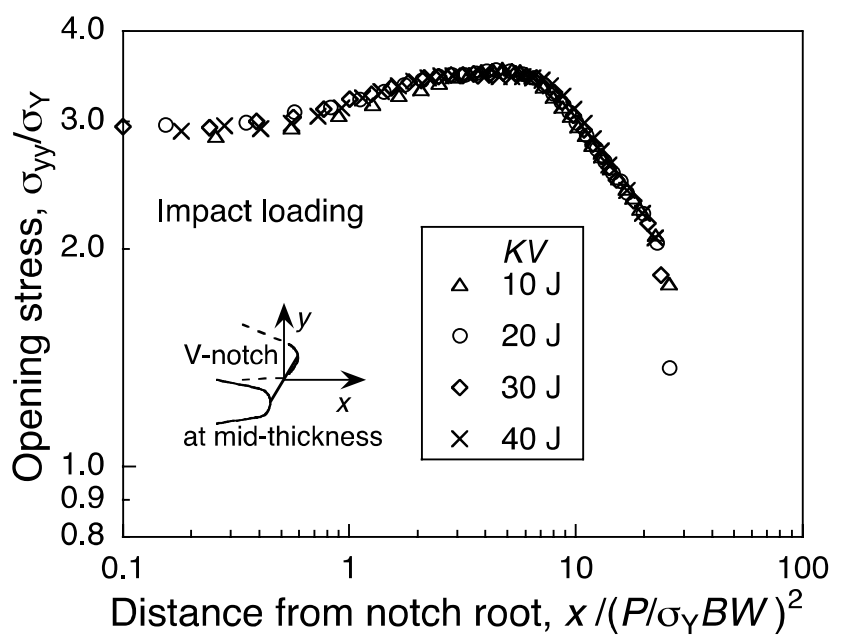

(a) $\sigma_{Y}=400 \mathrm{MPa}$

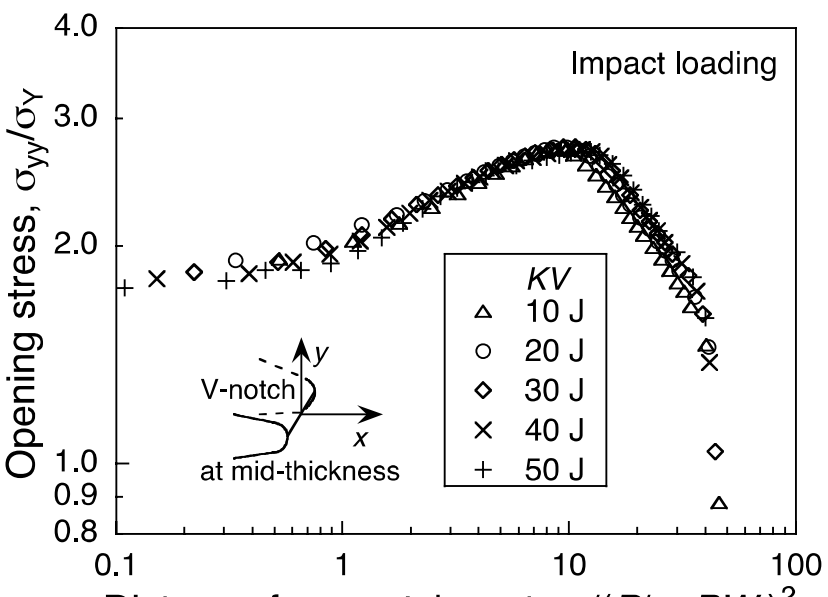

Distance from notch root, $x /\left(P / \sigma_{Y} B W\right)^{2}$

(b) $\sigma_{Y}=800 \mathrm{MPa}$

Fig. 5 Opening stress distribution near the V-notch in the Charpy specimen.

Subjected impact loading as a function of the parameter $x /\left(P / \sigma_{\mathrm{Y}} B W\right)^{2}$.

specimen of elastic-plastic material.

\section{Analysis of the Weibull Shape Parameter of Charpy Absorbed Energy}

In this study, the brittle fracture model, based on the weakest link theory, is proposed for evaluating the scatter in the Charpy absorbed energy.

In the local approach for cleavage fracture [4], the failure probability of a microcrack of critical length is

$$
p_{\mathrm{f}}\left(\sigma_{\mathrm{yy}}\right)=\left(\sigma_{\mathrm{yy}} / \sigma_{0}\right)^{m}
$$

where $\sigma_{0}$ and $m$ are material constants. In accordance with the weakest link theory, the specimen failure probability $F$ is given as follows:

$$
F=1-\prod_{i}\left(1-p_{f}\left(\sigma_{\mathrm{yy}, i}, V_{i}\right)\right) \simeq 1-\exp \left[-\sum_{\substack{\text { process zone } \\ i}} \frac{V_{i}}{V_{0}}\left(\frac{\sigma_{\mathrm{yy}, i}}{\sigma_{0}}\right)^{m}\right\rfloor
$$

where $\sigma_{\mathrm{yy}, i}$ is the opening stress in element $i, V_{\mathrm{i}}$ is the volume of element $i$, and $V_{0}$ is a normalization factor. Generally, the fracture process zone is considered to be the plastic zone for steels. According to Eq. 3, the exponential term in Eq. 5 is given as follows:

$$
\begin{aligned}
\sum_{\text {process zone }} \frac{V_{i}}{V_{0}} \sigma_{\mathrm{yy}}^{m} & =\frac{B}{V_{0}} \int_{-\pi}^{\pi} \int_{0}^{r_{p}(\theta)} \sigma_{\mathrm{yy}}^{m} r d r d \theta \\
& =\frac{B k^{m} \sigma_{\mathrm{Y}}^{m}}{V_{0}}\left(\frac{P}{\sigma_{\mathrm{Y}} B W}\right)^{4} \int_{-\pi}^{\pi} g^{m}(\theta) d \theta \int_{0}^{U_{p}(\theta)} f^{m}(U) U d U
\end{aligned}
$$

where $U$ is $r /\left(P / \sigma_{\mathrm{Y}} B W\right)^{2}$ and $r_{\mathrm{p}}(\theta)$ is $\left(k P g(\theta) / \sigma_{\mathrm{Y}} B W\right)^{2}$ and $U_{\mathrm{p}}(\theta)$ describes the plastic zone, i.e. $U_{\mathrm{p}}(\theta)$ $=k^{2} g^{2}(\theta)$. Consequently, Eq. 5 can be rewritten as

$$
F=1-\exp \left[-C\left(\frac{P^{2}}{\sigma_{0}^{2} B^{2} W^{2}}\right)^{2}\right]
$$

where $C=k^{m} \sigma_{\mathrm{Y}}^{m-4} \sigma_{0}^{m+2} B / V_{0} \int_{-\pi}^{\pi} g^{m}(\theta) d \theta \int_{0}^{U_{p}(\theta)} f^{m}(U) U d U$. 
The energy absorbed in the Charpy specimen is composed of elastic and plastic strain energy. Then, $K V$ is given as

$$
K V=W_{\mathrm{e}}+W_{\mathrm{p}}=\frac{P^{2}}{2 k}+W_{\mathrm{p}}
$$

where $W_{\mathrm{e}}$ and $W_{\mathrm{p}}$ are the elastic and plastic strain energy, respectively, and $k$ is the elastic loading slope. Fig. 6 shows the numerical results of the energy ratio $W_{\mathrm{p}} / K V$. When $K V$ is more than $10 \mathrm{~J}$, the ratio is approximately constant. Thus, Eq. 8 can be rewritten by assuming that $W_{\mathrm{p}}$ is $\beta K V$ :

$$
P^{2}=2 k\left(K V-W_{\mathrm{p}}\right)=2 k(1-\beta) K V
$$

where $\beta$ is constant.

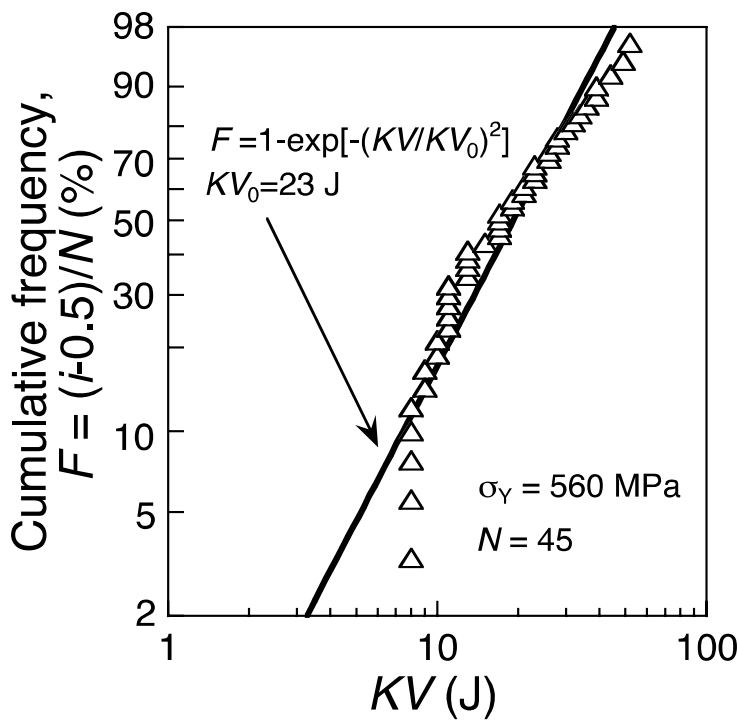

(a) HT590 steel

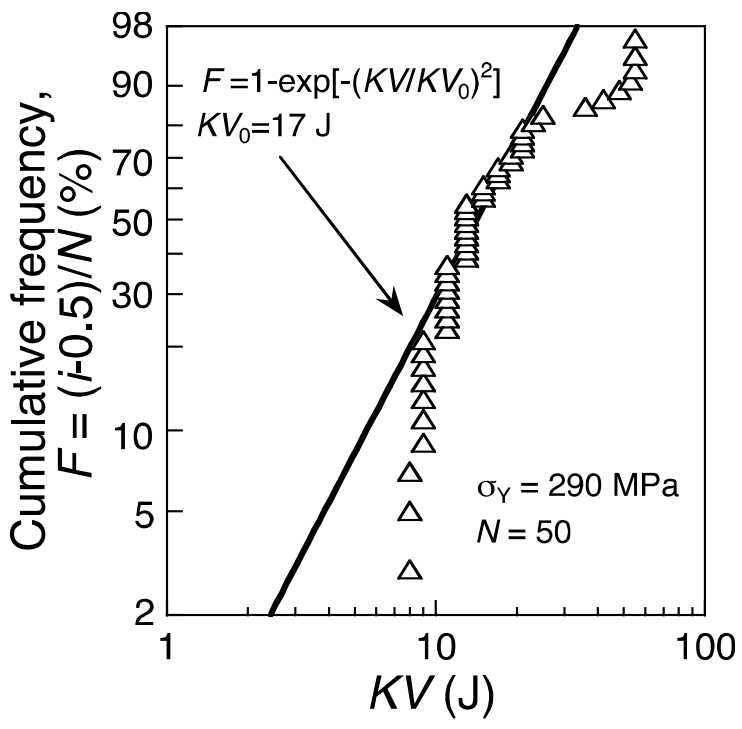

(c) Mild steel

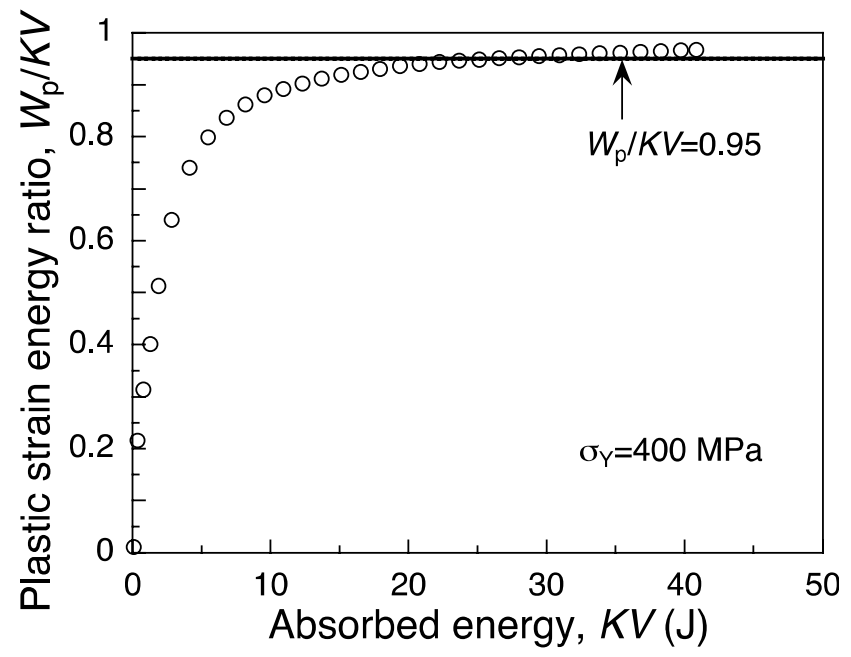

Fig. 6 Relationship between Charpy absorbed energy and plastic strain energy.

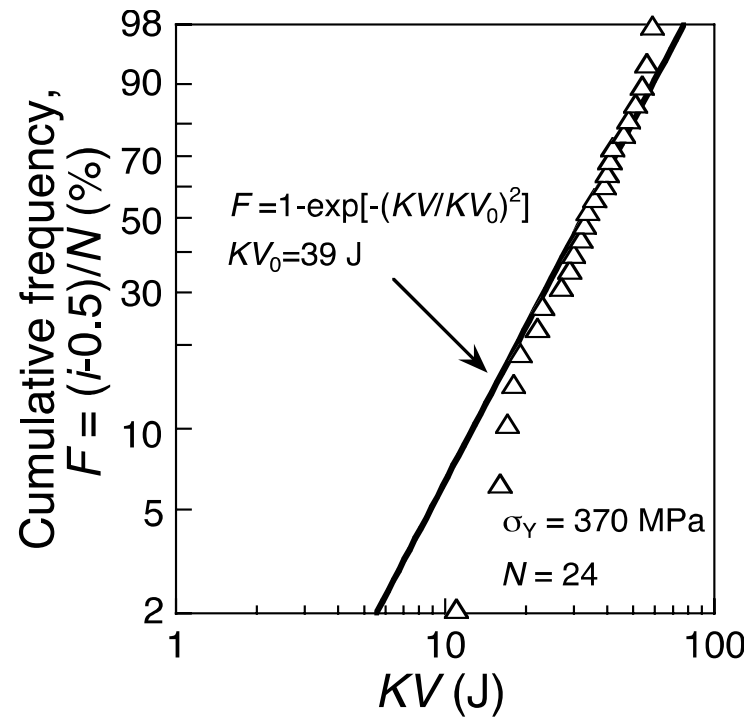

(b) HT490 stee

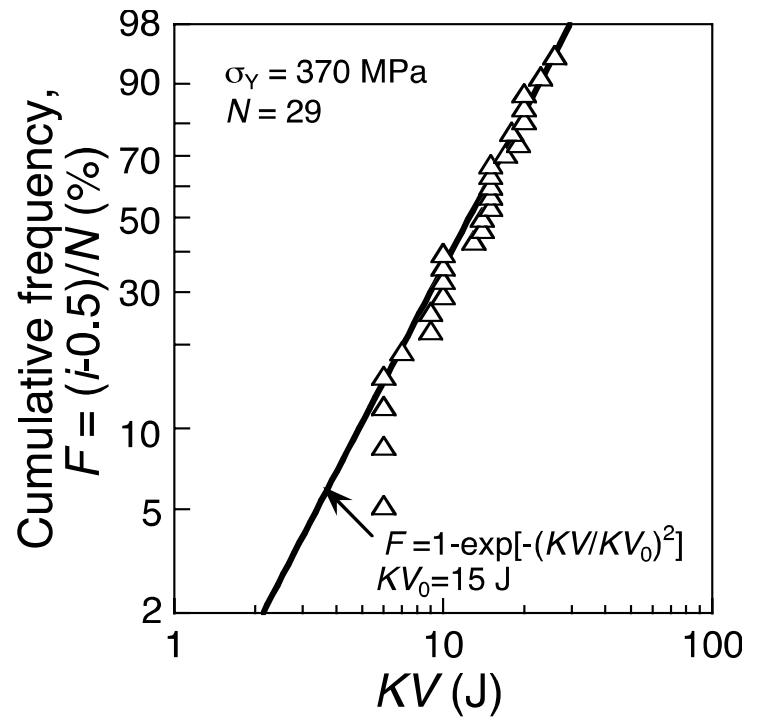

(d) Electrogas arc weld of HT490

Fig. 7 Weibull probability distribution of Charpy impact test results. 
Hence, from Eqs. 7 and 9, the failure probability at a certain $K V$ is given as follows:

$$
F=1-\exp \left[-\left(\frac{K V}{K V_{0}}\right)^{2}\right]
$$

where $K V_{0}$ is the scale parameter of the Weibull distribution and is equal to the value of $K V$ for a failure probability of $63.2 \%$.

Eq. 10 indicates that the Weibull shape parameter is 2 for Charpy absorbed energy. This statistical model is validated by the experimental results obtained for structural steels and welds in other studies $[2,6]$.

Kanazawa et al. [2] conducted a large number of Charpy tests on 590 MPa strength class (HT590) steel and a mild steel at the same temperature, which was lower than the DBTT. The Japan Ship Technology Research Association [6] conducted tests on $490 \mathrm{MPa}$ strength class (HT490) steel and an electrogas arc weld of the HT490 steel. The values of $K V$ obtained by the Charpy tests are shown in Fig. 7. The experimental results show a good fit to the regression line of the two-parameter Weibull distribution with the shape parameter of 2 .

These results validate that the statistical distribution of $K V$ at a lower temperature than the DBTT is characterized by the Weibull distribution. The shape parameter value for the $K V$ distribution is almost equal to 2 under the condition of pure brittle fracture. This finding leads to the statistical evaluation of the material toughness.

\section{Conclusions}

The stress fields ahead of the V-notch in a Charpy specimen were investigated by 3D FE analyses in terms of the stress singularity. The $1 / r^{1 / 2}$ singularity approximately dominates the stress field near the $\mathrm{V}$-notch. The amplitude of the opening stress fields ahead of the $\mathrm{V}$-notch in the Charpy specimen at varying amounts of $K V$ is uniquely described with the parameter $\left(P / \sigma_{\mathrm{Y}} B W\right)^{2}$.

With the numerical results, the brittle fracture model is proposed for evaluating the statistical scatter in the Charpy absorbed energy $K V$. It was found that the statistical distribution of $K V$ is characterized by a two-parameter Weibull distribution with the shape parameter of 2 under the condition of pure brittle fracture. This finding leads to the statistical evaluation of the material toughness in the Charpy V-notch impact test.

\section{References}

[1] M. Hirata, T. Ohwa: Journal of the Japan Welding Society, 32 (1963), pp. 450-454 (in Japanese).

[2] T. Kanazawa, S. Machida, and H. Yoshinari: Journal of the Society of Naval Architects of Japan, 150 (1981), pp. 532-540 (in Japanese).

[3] J. D. Landes and D. H. Shaffer: ASTM STP 700, Philadelphia (1980), pp. 368-382.

[4] F. M. Beremin: Metallurgical Trans. A, 14A (1983), pp. 2277-2287.

[5] K. Wallin: Engineering Fracture Mechanics, 19 (1984), pp. 1085-1093.

[6] Japan Ship Technology Research Association: SR153 research report (1975).

[7] T. Kubo, S. Igi, H. Shimanuki, M. Yamauchi, F. Minami, and M. Toyoda: Proceedings of the 7th International Symposium (2001), pp. 1129-1134.

[8] T. Nakamura, C. F. Shih, and L. B. Freund: Engineering Fracture Mechanics, 25 (1986), pp. 323-339. 